EXTENDED REPORT

\title{
Enhanced corneal compensation for scanning laser polarimetry on eyes with atypical polarisation pattern
}

\author{
M Tóth, G Holló
}

Br J Ophthalmol 2005;89:1139-1142. doi: 10.1136/bjo.2005.070011

See end of article for authors' affiliations

.....................

Correspondence to: Dr G Holló, Tömö u 25-29, 1083 Budapest, Hungary; hg@ szeml.sote.hu

Accepted for publication 2 May 2005

\begin{abstract}
Aim: To investigate the potential advantage of an enhanced corneal compensation algorithm (ECC) compared with variable corneal compensation (VCC) in the analysis of scanning laser polarimetric (SLP) images with atypical retardation pattern (ARP).

Methods: SLP-VCC images with ARP (typical scan score $($ TSS) $<80$ ) of one eye of each of 27 glaucoma patients and 19 healthy subjects were compared with the corresponding SLP-ECC images obtained at the same session.

Results: ARP was present in $10.4 \%$ of the normals and $15.5 \%$ of the glaucoma patients imaged with SLPVCC over 9 months. In both groups TSS was higher for ECC than for VCC $(p<0.001)$. In glaucoma TSNIT, superior and inferior average thickness values were significantly lower, and TSNIT (measuring ellipse around the optic nerve head in the four (temporal, superior, nasal, inferior) quadrants) standard deviation was significantly higher with ECC than with VCC $(p<0.001)$. In the normal group nerve fibre indicator (NFI) was lower with ECC than with VCC $(p=0.007)$. TSNIT average was smaller and TSNIT standard deviation was higher with ECC $(p<0.001)$. Superior and inferior average thickness did not differ between VCC and ECC in the normal group.

Conclusions: The new ECC software substantially improves polarimetric image analysis on eyes showing atypical polarisation pattern.
\end{abstract}

S canning laser polarimetry (SLP) with variable corneal compensation (VCC) has become a widely used noninvasive clinical method to measure the retinal nerve fibre layer thickness (RNFLT) and to detect glaucoma. ${ }^{1-5}$ As VCC compensates for the true anterior segment retardation, measurements with SLP-VCC are more exact than those obtainable with the earlier instrument which had a fixed corneal polarisation compensator. ${ }^{1-5}$

VCC measurements, however, sometimes exhibit an atypical retardation pattern (ARP), which makes interpretation of the results difficult or impossible. ${ }^{67} \mathrm{ARP}$, caused by poor signal to noise ratio, is characterised by irregular patches of elevated retardation values which do not match the expected retardation distribution based on the retinal nerve fibre layer..$^{6-9}$ It is not infrequent among myopic eyes and occurs also in a proportion of emmetropic eyes. Eyes showing ARP have not been considered optimal candidates for SLP, and are usually excluded from the analysis. ${ }^{6-8}$

To improve the signal to noise ratio and yet still achieve individualised corneal compensation, a new software based compensation method, called enhanced corneal compensation algorithm (ECC), has been developed. ${ }^{9}$ In ECC, a known large birefringence bias is introduced into the measurement beam path to shift the measurement of total retardation into a higher value region; this is in contrast to VCC measurement where SLP directly measures the relatively low RNFL retardation. The birefringence bias is determined from the macular region of each measurement and then, point by point, removed mathematically to yield the true RNFL retardation.

In order to investigate the potential benefit of ECC over VCC in human eyes with ARP, we compared corresponding SLP-VCC and SLP-ECC images of healthy and glaucomatous eyes, which all showed ARP when examined using the commercially available VCC compensation method.

\section{METHODS}

The research protocol was approved by the Institutional Review Board for Human Research of the Semmelweis University, and informed consent was obtained from all participants before imaging was done. Of the 357 White individuals ( 174 glaucoma patients and 183 healthy subjects) imaged with SLP-VCC between May 2004 and January 2005, 27 glaucoma patients (mean age 68.4 years, range 42-82 years) suffering from chronic open angle glaucoma (primary open angle, normal tension, or juvenile glaucoma) and 19 healthy White subjects (mean age 44.8 years, range 18-80 years) without any ocular abnormality were enrolled in the study. The glaucoma patients had reliable and reproducible visual field defects typical for glaucoma, shown with the Octopus program G2 threshold perimetry test (MD and CPSD higher than $2 \mathrm{~dB}$ with inferior and/or superior paracentral or arcuate scotomas, nasal step, hemifield defect, or generalised depression) as well as glaucomatous and optic nerve head damage (diffuse or localised neuroretinal rim thinning, evaluated stereoscopically), and open anterior chamber angle on both eyes. Each subject in the healthy group had reliable and normal visual field, normal optic nerve head, and normal ophthalmic status for both eyes. Each participant had sufficient central vision for optimal fixation, in both eyes.

Scanning laser polarimetry was performed by the same investigator (MT) without pupil dilation using the GDx VCC instrument (Laser Diagnostic Technologies, Inc, San Diego, CA, USA) with software version 5.4.0.27. The instrument works both with VCC and, in a special research mode version,

Abbreviations: ARP, atypical retardation pattern; ECC, enhanced corneal compensation; RNFLT, retinal nerve fibre layer thickness; SLP, scanning laser polarimetry; TSNIT, measuring ellipse around the optic nerve head in the four (temporal, superior, nasal, inferior) quadrants; TSS, typical scan score; VCC, variable corneal compensation. 

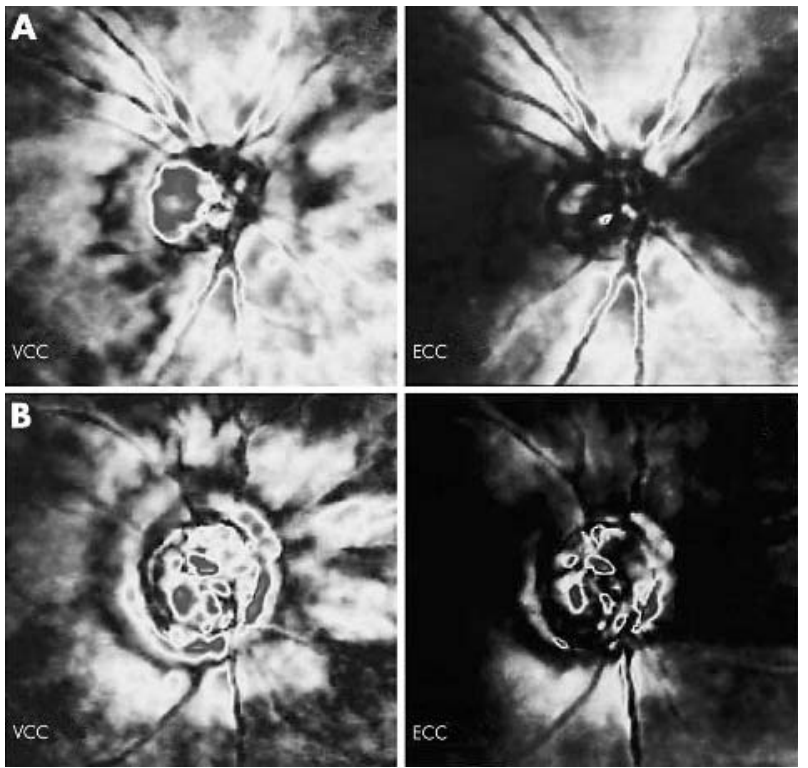

Figure 1 Two examples for the neutralisation of clinically significant atypical retardation pattern by the enhanced corneal compensation (ECC) software. (A) A healthy eye (typical scan score (TSS) value: 30 with variable corneal compensation (VCC), 100 with ECC; nerve fibre indicator (NFI): 14 with VCC, 13 with ECC), (B) A glaucomatous eye (TSS value: 15 with VCC, 94 with ECC; NFI: 28 with VCC, 75 with ECC)

with ECC. To stabilise fixation, an internal fixation stimulus was used. The cornea retardation was calculated for each eye with each type of compensation. This calculation is accomplished by scanning the eye without any compensation. The macular region of the uncompensated image is analysed to determine the slow cornea axis and magnitude of birefringence for the specific eye. This method has been described in detail elsewhere. ${ }^{10}$ In brief, the method is based on the assumption of uniform birefringence around the macula, where it is due to the Henle's fibre layer. The non-uniform birefringence pattern in the macula can be used to calculate the axis and magnitude of birefringence due to the cornea. In the ECC mode the compensator is adjusted so that it combines with the corneal retardation to produce a bias retardation of approximately $55 \mathrm{~nm}$ and a slow axis to be close to vertical. The SLP then measures a higher total retardation than the RNFL retardation alone, and the signal to noise ratio is improved as a result. The actual bias retardation and axis in each image are measured from the macular region, in a similar way to corneal birefringence measurement. The actual retinal nerve fibre layer retardation is derived mathematically. The actual bias is determined from each image, and removed from the final RNFL image. ${ }^{9}$ This new ECC method has been described at the recent ISIE meeting by Dr Zhou of Laser Diagnostic Technologies (ISIE, 2004).

In our investigation, SLP measurements using both the VCC and the ECC technique were performed on each eye at the same session. Actual ametropia was corrected for before image acquisition, using the software of the instrument. The optic nerve head ellipse was placed by the operator on the edge of the disc. All RNFLT measurements were based on a fixed size measurement circle of diameter $3.2 \mathrm{~mm}$ which is centred on the operator placed ellipse. Presence of ARP (fig 1) on the peripapillary SLP-VCC image was assessed subjectively (in masked fashion) by one investigator $(\mathrm{GH})$, and also by using the software provided typical scan score (TSS), which automatically calculates the degree of typicality between 0 (maximally atypical pattern) and 100 (completely typical
Table 1 Definition of the parameters analysed in this study

\begin{tabular}{|c|c|}
\hline $\begin{array}{l}\text { Polarimetric } \\
\text { parameter }\end{array}$ & Definition \\
\hline $\begin{array}{l}\text { TSNIT average } \\
\text { thickness }\end{array}$ & $\begin{array}{l}\text { Average thickness }(\mu \mathrm{m}) \text { for the pixels in the } \\
\text { temporal }(T) \text {, superior }(S) \text {, nasal }(N) \text {, and inferior } \\
\text { (I) quadrants along the total measured ring }\end{array}$ \\
\hline $\begin{array}{l}\text { TSNIT standard } \\
\text { deviation }\end{array}$ & $\begin{array}{l}\text { The standard deviation }(\mu \mathrm{m}) \text { of the retinal nerve } \\
\text { fibre layer thickness (RNFLT) values in the } \\
\text { temporal (T), superior (S), nasal (N), and inferior } \\
\text { (I) quadrants along the measured ring }\end{array}$ \\
\hline Superior average & $\begin{array}{l}\text { The average RNFLT }(\mu \mathrm{m}) \text { of the points along the } \\
\text { superior } 120^{\circ} \text { section of the ring }\end{array}$ \\
\hline Inferior average & $\begin{array}{l}\text { The average RNFLT }(\mu \mathrm{m}) \text { of the points along the } \\
\text { inferior } 120^{\circ} \text { section of the ring }\end{array}$ \\
\hline NFI & $\begin{array}{l}\text { Nerve fibre indicator. An indicator of the } \\
\text { probability that the eye suffers from glaucoma. It } \\
\text { is generated by neural network technology, } \\
\text { trained with data from normal to glaucomatous } \\
\text { eyes. It represents a single, summary measure of } \\
\text { the integrity of the entire RNFLT }\end{array}$ \\
\hline
\end{tabular}

pattern). TSS is based on a support vector machine analysis, and is applicable to both VCC and ECC images. TSS is based on the slope, standard deviation, and average magnitude of the measured thickness extending from the edge of the optic nerve head outward to 20 degrees. The parameters are calculated separately for each quadrant and along the wedge in each quadrant. ARP was defined as non-RNFLT based peripapillary polarisation, with TSS value $<80$. Each participant had ARP and a TSS value $<80$ for at least one eye. Both eyes were imaged but only one eye per participant, the eye with the more atypical ARP (that is, with lower TSS value) as found with VCC was included in the analysis. The quality of each image included in the study was optimal (quality score $\geqslant 8$ ). Ten eyes were hyperopic and 25 myopic, but only three of the latter group had myopia $\geqslant-7.0 \mathrm{D}$. None of the eyes had a tilted optic nerve head. The SLP parameters analysed are defined in table 1. Each parameter is automatically calculated, both for the VCC and the ECC reports.

\section{Statistics}

The SPSS 10.0 program package was used for statistical analysis (SPSS Inc, Chicago, IL, USA). The Wilcoxon signed rank test was used to compare NFI and TSS, and the paired $t$ test was used to compare TSNIT average, TSNIT standard deviation, superior average, and inferior average between VCC and ECC within the subject groups. The Mann-Whitney $\mathrm{U}$ test was used for comparison of TSS between the two subject groups. Pearson's correlation test was used to investigate correlation between the TSS values and the VCC/ECC difference of the TSNIT, superior, and inferior average thickness values, respectively. p Values of less than 0.05 were considered statistically significant.

\section{RESULTS}

Atypical retardation pattern was found in 19 of the 183 healthy subjects $(10.4 \%)$ and in 27 of the 174 glaucoma patients $(15.5 \%)$ imaged with SLP-VCC over a period of 9 months.

All images selected for this study showed clinically significant ARP with VCC, which decreased considerably when the ECC software was used (fig 1). Tables 2 and 3 show the corresponding values determined with VCC and ECC for each parameter within each subject group. In both groups TSS was significantly higher with ECC than when using VCC $(\mathrm{p}<0.001)$. 
Table 2 Influence of the type of corneal compensation (variable (VCC) $v$ enhanced (ECC)) on typical scan score (TSS) and nerve fibre indicator (NFI) of images showing atypical retardation pattern

\begin{tabular}{|c|c|c|c|c|c|c|c|}
\hline \multirow[b]{2}{*}{ Parameter } & \multicolumn{3}{|l|}{ VCC } & \multicolumn{3}{|l|}{ ECC } & \multirow[b]{2}{*}{ p Value* } \\
\hline & r Percentile 25 & Median & Percentile 75 & Percentile 25 & Median & Percentile 75 & \\
\hline TSS $\dagger$ & 23 & 46 & 61 & 90 & 100 & 100 & $<0.001$ \\
\hline TSS $\ddagger$ & 39 & 53 & 68 & 98 & 100 & 100 & $<0.001$ \\
\hline NFI† & 22 & 29 & 60 & 23 & 46 & 62 & 0.069 \\
\hline NFIł & 15 & 21 & 33 & 12 & 20 & 22 & 0.007 \\
\hline \multicolumn{8}{|c|}{$\begin{array}{l}\text { *Wilcoxon signed ranks test. } \\
\text { †Glaucoma group. } \\
\text { ‡Normal group. }\end{array}$} \\
\hline
\end{tabular}

In the glaucoma group TSNIT, superior, and inferior average thickness were significantly lower. TSNIT standard deviation was significantly higher with ECC than with VCC $(\mathrm{p}<0.001)$. NFI did not show a significant difference between the two compensation techniques.

In the normal group TSNIT average was smaller, and TSNIT standard deviation was higher with ECC $(p<0.001)$, but superior and inferior average thickness did not differ between VCC and ECC. NFI was significantly lower with ECC than with VCC $(p=0.007)$.

The VCC/ECC difference of TSNIT, superior, and inferior average thickness values showed a significant negative correlation with VCC-TSS $(r-0.660,-0.579$, and -0.434 , respectively; $\mathrm{p}<0.004$ for each correlation), but not with ECC-TSS ( $p>0.05$ for each correlation). TSS did not differ between the glaucoma patients and the healthy subjects either with VCC (40.2 (SD 24.2) v $51.4($ SD 19.7); $\mathrm{p}=0.141)$ or ECC $(94.9$ (8.0) $v 97.3$ (SD 6.2); $\mathrm{p}=0.354)$.

\section{DISCUSSION}

In our investigation, ECC, a new corneal compensation software technique for SLP was investigated in a comparison with the presently available VCC technique, on glaucomatous and normal eyes with ARP. It is well known from clinical practice that ARP is not optimally neutralised with the commercially available VCC software. ${ }^{11}$ Therefore eyes showing atypical retardation are usually excluded from clinical SLP measurements and polarimetric research. ${ }^{8}$ In a recent study ARP was found in $25 \%$ of the normal eyes and $51 \%$ of the glaucomatous ones. ${ }^{11}$ The ECC method was developed in order to improve neutralisation of atypical polarimetric artefacts, and to increase the dynamic range of the measurements in the low signal range. ${ }^{9}$ As ECC is a software development and requires no hardware changes, it has the potential to be widely used in clinical practice on the available SLP instruments, if the method proves to be more useful than VCC.

An important aspect of our study was to evaluate the effect of ECC on the typicality of the peripapillary SLP image of eyes showing ARP with VCC. Table 2 shows that TSS improved significantly when ECC was used. This improvement was also significant from a clinical point of view, because the score moved very close to the ideal 100, and the image quality improved considerably in both groups, as illustrated in figure 1. Another important question is whether ECC influences images taken from glaucomatous and normal eyes in a similar way. By comparing the TSS values between the subject groups we found no difference with either compensation method. This suggests that the effect of ECC on the typicality is not influenced by the presence of glaucoma.

The influence of ECC on the measured SLP values was also investigated in our study. In another, unpublished study (Holló G, Tóth M. IOVS 2005;46:ARVO E-abstract 2500) we found that the values do not differ between VCC and ECC if the images contain no ARP. This is supported by the significant negative correlation found between VCC-TSS and the VCC/ECC thickness difference in the present study, which means that the more typical the image, the smaller the difference between the corresponding values obtained with the two compensation methods. In contrast, the lack of correlation with ECC-TSS suggests that in the ECC mode the measured RNFLT is not influenced by the magnitude of ARP.

The results of this on eyes with ARP show that when using ECC the retinal polarisation became more real; in the glaucoma group neutralisation of atypical retardation with ECC resulted in lower thickness values and increased TSNIT standard deviation. In contrast, in the healthy group the superior and inferior average thickness values did not differ between VCC and ECC. This shows that in the healthy eyes neutralisation of ARP did not diminish the true thickness

Table 3 Influence of the type of corneal compensation (variable (VCC) $v$ enhanced (ECC)) on the measured values on images showing atypical retardation pattern

\begin{tabular}{|c|c|c|c|c|c|}
\hline Parameter & VCC mean (SD) & ECC mean (SD) & $\begin{array}{l}\text { Difference of the } \\
\text { mean (SD) }\end{array}$ & $\begin{array}{l}95 \% \mathrm{Cl} \text { for the } \\
\text { difference }\end{array}$ & p Value* \\
\hline $\begin{array}{l}\text { TSNIT average } \\
\text { TSNIT average } \\
\text { TSNIT SD† } \\
\text { TSNIT SD } \ddagger \\
\text { Superior average } † \\
\text { Superior average } \ddagger \\
\text { Inferior average† } \\
\text { Inferior average } \neq\end{array}$ & $\begin{array}{l}53.4(9.6) \\
58.2(6.3) \\
15.1(4.3) \\
19.0(4.1) \\
56.4(12.0) \\
65.0(9.8) \\
59.7(10.5) \\
67.3(9.9)\end{array}$ & $\begin{array}{l}42.7(7.9) \\
51.8(6.1) \\
18.9(4.5) \\
25.1(4.5) \\
49.5(12.8) \\
64.3(11.3) \\
53.6(10.4) \\
66.2(8.4)\end{array}$ & $\begin{array}{r}10.7(6.2) \\
6.4(3.6) \\
-3.8(3.7) \\
-6.1(3.1) \\
6.9(7.7) \\
0.7(5.0) \\
6.1(5.2) \\
1.1(4.3)\end{array}$ & $\begin{array}{l}8.3 \text { to } 13.2 \\
4.7 \text { to } 8.2 \\
-5.3 \text { to }-2.3 \\
-7.7 \text { to }-4.6 \\
3.9 \text { to } 9.9 \\
-1.8 \text { to } 3.1 \\
4.0 \text { to } 8.1 \\
-0.9 \text { to } 3.2\end{array}$ & $\begin{array}{r}<0.001 \\
<0.001 \\
<0.001 \\
<0.001 \\
<0.001 \\
0.572 \\
<0.001 \\
0.260\end{array}$ \\
\hline \multicolumn{6}{|l|}{$\begin{array}{l}\text { *Paired } t \text { test. } \\
\text { †Glaucoma group. } \\
\text { †Normal group. } \\
\text { TSNIT SD TSNIT star }\end{array}$} \\
\hline
\end{tabular}


data. As ARP became neutralised on the temporal and nasal peripapillary areas, the TSNIT average thickness decreased and the TSNIT standard deviation increased. As a result of these differences the RNFLT became even more normal, and the NFI value decreased significantly. This shows that the normal eyes with ARP are in fact healthier than is indicated with the commercially available VCC software.

In conclusion, the new ECC compensation software for SLP reduced atypical polarisation signals in a clinically and statistically significant manner in our subjects, who all showed ARP with the commercially available VCC technique. These subjects represented $10.4 \%$ of the normals and $15.5 \%$ of the glaucoma patients imaged with SLP over 9 months, which suggests that ECC may have an important role in polarimetry in future.

\section{ACKNOWLEDGEMENTS}

The authors thank Péter Vargha for assistance with the data analysis, and Qienyuan Zhou for providing information on the ECC software.

\section{Authors' affiliations}

M Tóth, G Holló, Department of Ophthalmology, Semmelweis University, Budapest, Hungary

Sponsorship: supported in part by Hungarian National Health Grant (ETT) 011/2003 (Dr Holló).

Competing interests: Dr Holló has previously acted as an unpaid consultant of Laser Diagnostic Technologies, Inc. Dr Tóth has no competing interest.

Ethics approval (see also in the Methods section): the research protocol was approved by the Institutional Review Board for Human Research of the Semmelweis University, and informed consent was obtained from all participants before imaging was done.

\section{REFERENCES}

1 Weinreb RN, Bowd C, Zangwill LM. Glaucoma detection using scanning laser polarimetry with variable corneal polarization compensation. Arch Ophthalmol 2003;121:218-24.

2 Choplin NT, Zhou Q, Knighton RW. Effect of individualised compensation for anterior segment birefringence on retinal nerve fiber layer assessments as determined by scanning laser polarimetry. Ophthalmology 2003;110:719-25.

3 Greenfield DS, Knighton RW, Fever WJ, et al. Correction for corneal polarization axis improves the discriminating power of scanning laser polarimetry. Am J Ophthalmol 2002;134:27-33.

4 Tannenbaum DP, Hoffmann D, Lemij HG, et al. Variable corneal compensation improves discrimination between normal and glaucomatous eyes with the scanning laser polarimeter. Ophthalmology 2004;111:259-64.

5 Colen TP, Tang NE, Mulder PG, et al. Sensitivity and specificity of new GDx parameters. J Glaucoma 2004;13:28-33.

6 Hoh ST, Greenfield DS, Liebmann JM, et al. Factors affecting image acquisition during scanning laser polarimetry. Ophthalmic Surg Lasers 1998:29:545-51.

7 Bozkurt B, Irkec M, Gedik S, et al. Effect of peripapillary chorioretinal atrophy on GDx parameters in patients with degenerative myopia. Clin Experiment Ophthalmol 2002;30:411-14.

8 Medeiros FA, Zangwill LM, Bowd C, et al. Comparison of the GDx VCC scanning laser polarimeter, HRT II confocal scanning laser ophthalmoscope, and Stratus OCT optical coherence tomography for the detection of glaucoma. Arch Ophthalmol 2004;122:827-37.

9 Zhou $\mathbf{Q}$. Retinal scanning laser polarimetry and methods to compensate for corneal birefringence. The New Optics of the Human Eye 2005 (in press).

10 Zhou $Q$, Weinreb RN. Individualized compensation of anterior segment birefringence during scanning laser polarimetry. Invest Ophthalmol Vis Sci 2002;43:2221-8.

11 Bagga H, Greenfield DS, Feuer WJ. Quantitative assessment of atypical birefringence images using scanning laser polarimetry with variable corneal compensation. Am J Ophthalmol 2005; 139:437-46. 